\title{
In silico single polymorphism analysis of RPMS1 and A73 gene in Nasopharyngeal Carcinoma
}

\author{
Quang Trong Minh ${ }^{1}$, Nguyen Hoang Danh ${ }^{1}$, Thieu Hong Hue ${ }^{2}$, Lao Duc Thuan ${ }^{1,2 *}$ \\ ${ }^{1}$ Ho Chi Minh City Open University, Vietnam \\ ${ }^{2}$ Vietnam National University Ho Chi Minh City, Vietnam \\ *Corresponding author: thuan.ld@ ou.edu.vn
}

\begin{tabular}{|c|c|}
\hline ARTICLE INFO & ABSTRACT \\
\hline $\begin{array}{l}\text { DOI: } 10.46223 / \mathrm{HCMCOUJS} . \\
\text { tech.en.9.1.348.2019 }\end{array}$ & $\begin{array}{l}\text { Nasopharyngeal Carcinoma (NPC) is one of the most } \\
\text { frequent cancer types in Vietnam, with a high mortality rate. } \\
\text { Therefore, the early diagnosis and detection of NPC are urgently } \\
\text { needed to improve patient survival. Recent studies have confirmed } \\
\text { that the infection of Epstein-Barr Virus (EBV) and polymorphism } \\
\text { in RPMS1 and } A 73 \text { are considered as the etiological factor } \\
\text { associated with NPC. However, in Vietnam, there are no studies } \\
\text { relevant to the identification of polymorphism of RMPS1 and } A 73 \text {. }\end{array}$ \\
\hline Received: April 21 ${ }^{\text {st }}, 2019$ & $\begin{array}{l}\text { With the aims, in future, to develop a technique based on detecting } \\
\text { the frequencies of } R P M S 1 \text { and } A 73 \text { variants as biomarkers of }\end{array}$ \\
\hline Revised: May $10^{\text {th }}, 2019$ & prognosis and early diagnosis of NPC, we conducted the initial in \\
\hline Accepted: May 21 st 2019 & $\begin{array}{l}\text { silico analysis (1) Data collection, statistical analysis the } \\
\text { frequencies detection of } R P M S 1 \text { and } A 73 \text { variants from various } \\
\text { previous published studies; (2) Determine experimental methods } \\
\text { to predict and diagnose early NPC and examine necessary steps in } \\
\text { silico. As the results, we established the systematic databases of } \\
\text { RPMSI and } A 73 \text { polymorphism, and evaluation the primer set for }\end{array}$ \\
\hline Keywords: & $\begin{array}{l}\text { the amplification of } R P M S 1 \text { and } A 73 \text {, which could be applied in } \\
\text { further studies related to the identification of } R P M S 1 \text { and } A 73\end{array}$ \\
\hline $\begin{array}{l}\text { A73, NPC, polymorphism, } \\
\text { RPMS1 }\end{array}$ & $\begin{array}{l}\text { gene polymorphism to find out the potential biomarkers for } \\
\text { screening, diagnosis as well as NPC treatment. }\end{array}$ \\
\hline
\end{tabular}

\section{Introduction}

Nasopharyngeal carcinoma (NPC), which has a striking geographic and ethnic distribution, has been considered as the most common and highly incident cancer of the head and neck cancer in Asian countries, especially in Vietnam (Regaud, 1921; Schmincke, 1921). According to GLOBOCAN (2018), the high prevalence of NPC cases was observed in reached to 164,671 cases $(\mathrm{ASR}=5.7 / 100,000)$ and deaths were 114,871 cases $(\mathrm{ASR}=3.9 / 100,000)$ in Vietnamese population. Notably, the symptoms of NPC were unclear such as hearing loss, nosebleeds, headache, feeling of fullness in the ear, etc., thus, NPC often presents at an advanced stage when first diagnosis (stage 3 or 4). Therefore, there is a challenge for finding an early diagnosis and biomarker to achieve favorable treatment and increase patient survival. 
Up to date, many studies have been demonstrated that multiple etiological factors, including Epstein-Barr virus (EBV) infection, genetics/or genetic susceptibility and epigenetics factors, have been suggested to be strongly linked with NPC (Hildesheim \& Levine, 1993). Moreover, there is growing evidence demonstrating that many Single Nucleotide Polymorphisms (SNPs) in EBV, including RPMS1 and A73, may have associated with NPC risk through the contribution to many cell signal pathways, such as cell-cycle regulation, apoptosis, cell differentiation, ... (Brooks, Lear, Young, \& Rickinson, 1993; Li et al., 2005; Li, Zhao, Hu, Zhao, \& Zhang, 2006).

RPMS1 gene belongs to the BamHI-A Rightward Transcripts (BARTs) family, starts at position 138,325 and ends at position 160,531 on the wild EBV gene (de Jesus et al., 2003; Sadler \& Raab-Traub, 1995; Smith et al., 2000). Up to now, a number of studies have been demonstrated that SNPs of RPMS1 G155391A, was specific to NPC, based on the amino acid substitution at position 155391 (Genbank, NC_007605). Its substitution resulted in a decrease in the degradation of the oncogenic RPMS1 protein. (Cui et al., 2017; Feng et al., 2015; Wu et al., 2018). According to Wu et al. (2018), they observed four significant variants: G155325T, G155326A, C155389T and G155391A, on the RPMS1 gene, and classified them as the four subtypes of RPMS1: RPMS1-A, RPMS1-B, RPMS1-C, and RPMS1-D. Among them, RPMS1C, RPMS1-D was reported to be strongly associated with NPC tumor formation. (Wu et al., 2018). A73 (also called RB2), one of the primary members of the EBV BARTs family, is reported to be related to NPC tumorigenesis (Zhang et al., 2007). The study indicated that A157154C in $A 73$ gene was detected almost exclusively in Chinese populations and preferentially exists in biopsies of nasopharyngeal carcinoma ( $\mathrm{Li}$ et al., 2006). Not only that, three polymorphisms A157154C, G159188C, and G159209C were also identified in A73 gene by Zhang et al. (2007). Among them, A157154C showed high frequency and might be correlated to the occurrence and development of NPC (Shen et al., 2015; Zhang et al., 2007).

In Vietnam, there is still no research about the variants of RPMS1 and $A 73$ in NPC, thus, in the current report, we summarized the current evidence about the polymorphism of $R P M S 1$ and $A 73$ in NPC based on the various previous publication via a systematic literature revision. The identification of SNPs of these genes may serve as targets for our further experiment and might become a potential biomarker for NPC in Vietnamese population.

\section{Materials and methods}

\subsection{Literature analysis, eligibility criteria and data extraction}

A systematic literature analysis was conducted based on the comprehensive search of observational studies. Previous studies were obtained from the following databases using validated search strategies: PubMed, Web of Science databases, Embase Database, etc. The following keywords were used: Nasopharyngeal carcinoma, A73, RPMS1, single nucleotide polymorphism, etc.; conducted up to April 2019. For these articles fulfilling the following criteria were used in the systematic review. Inclusion criteria: [1] Case-control study or cohort study that published the SNPs of RPMS1 and $A 73$ gene profile. [2] Studies that investigated SNPs of RPMS1 and $A 73$ in NPC. [3] Study that mention detection, the analysis method of SNPs of RPMS1 and A73. 
Using these articles, we extracted the frequency of detection RPMS1 and A73 variants, country, method, etc. When data were available, we reported the ranged and computed average weight frequencies of the candidate gene in studies.

\subsection{Evaluation primer for PCR-sequencing}

The sequence and information of RPMS1 and A73 gene were downloaded from Genbank (https://www.ncbi.nlm.nih.gov/genbank/) with accession number NC_007605, V01555 respectively. For evaluation of PCR primers, primer's physical characteristics were computed by IDT OligoAnalyzer 3.1 (http://sg.idtdna.com/calc/analyzer), Annhyb and primer-BLAST (https://www.ncbi.nlm.nih.gov/tools/primer -

blast/index.cgi?LINK_LOC=BlastHome) were tools for finding specific primers, as well as PCR product length.

\subsection{Building phylogenetic tree}

For the RPMS1 and A73 databases, the variants' sequences were collected from Genbank (NCBI) based on the previous publications. The relationship between RPMS1 subtypes, as well as $A 73$ was evaluated by Phylogenetic Tree was built by MEGA6 software based on using Joining Neural algorithm, the optimal evolutionary model was Jukes-Cantor and bootstrap values repeated 1000 times.

\section{Results}

\subsection{Database of variants in RPMS1 and A73 gene}

Up to April 2018, there were only three published studies, investigated SNPs of RPMS1. According to these studies, RPMS1 was classified into four subtypes: RPMS1-A, RPMS1-B, RPMS1-C, RPMS1-D (Wu et al., 2018). In particular, RPMS1-A includes random mutants in positions from nt 155277 - 155546 of RPMS1 gene, for example G155284T, G155307T, C155358T, C155481A, C155525T, A155545G (Wu et al., 2018). The RPMS1-B consisted of a mutant in the nucleotides G155377A (RPMS1-B1) and C155389T (RPMS1-B2) (Wu et al., 2018). The RPMS1-C included mutant in position G155391A (Wu et al., 2018. RPMS1-D variant encompassed mutant position G155325T and G155326A (Wu et al., 2018) (Table 1).

By using many keywords described above, a systematic literature analysis was conducted based on the comprehensive search of $A 73$ variant studies. As result, only one polymorphism of $A 73$ included a characteristic variant was $A 157145 \mathrm{C}$, related to NPC (Han, 2013; Li et al., 2006; Shen et al., 2015; Zhang et al., 2007). All polymorphism studies in the A73 gene included: Li et al. (2006); Han (2013); Zhang et al. (2007); Shen et al. (2015), certainly definite only polymorphism point A157145C was significantly associated in NPC. This variant was located on exon $\mathrm{V}$ in the $A 73$ gene also in the $A 73$ coding region but did not cause any amino acid alteration in protein A73 (CCA: Pro $\rightarrow$ CCC: Pro) (Table 1).

\section{Table 1}

Location of variants in the gene RPMS1 and $A 73$ in NPC

\begin{tabular}{ccccc}
\hline Variants & Positions & $\begin{array}{c}\text { Changed } \\
\text { nucleotide }\end{array}$ & $\begin{array}{c}\text { Changed amino } \\
\text { acid }\end{array}$ \\
\hline RPMS1 & RPMS1-A & $*$ & - & $*$ \\
RPMS1-B1 & 155377 & $\mathrm{G} \rightarrow \mathrm{A}$ & $\mathrm{S} \rightarrow \mathrm{N}$
\end{tabular}




\begin{tabular}{ccccc}
\hline Variants & Positions & $\begin{array}{c}\text { Changed } \\
\text { nucleotide }\end{array}$ & $\begin{array}{c}\text { Changed amino } \\
\text { acid }\end{array}$ \\
\hline RPMS1-B2 & 155389 & $\mathrm{C} \rightarrow \mathrm{T}$ & $\mathrm{P} \rightarrow \mathrm{L}$ \\
& RPMS1-C & 155391 & $\mathrm{G} \rightarrow \mathrm{A}$ & $\mathrm{D} \rightarrow \mathrm{N}$ \\
& RPMS1-D & 155325 & $\mathrm{G} \rightarrow \mathrm{T}$ & $\mathrm{G} \rightarrow$ Stop codon \\
\hline A73 & A157154C & 155326 & $\mathrm{G} \rightarrow \mathrm{A}$ & Not change \\
\hline
\end{tabular}

Note: “*” The changed nucleotides were randomized in the RPMS1 gene's region (from nucleotide 155277 to 155546), except for nucleotides positions of the RPMS1-B, C, D

Source: The researcher's data analysis

\subsection{Characteristics of included studies}

Overall, a total of three previously published studies were identified, accessed for eligibility from inclusion criteria, enrolled into systematic revision by using keywords described above. The characteristics of those studies were shown in Table 2.

\section{Table 2}

Studies included in the systematic review about RPMS1 variants in NPC

\begin{tabular}{|c|c|c|c|c|c|c|c|c|c|c|}
\hline \multirow{2}{*}{ Studies } & \multirow{2}{*}{ SCS } & \multirow{2}{*}{ SC } & \multicolumn{2}{|c|}{ RPMS1-A } & \multicolumn{2}{|c|}{ RPMS1-B } & \multicolumn{2}{|c|}{ RPMS1-C } & \multicolumn{2}{|c|}{ RPMS1-D } \\
\hline & & & Case & Control & Case & Control & Case & Control & Case & Control \\
\hline $\begin{array}{l}\text { Wu et al. } \\
\text { (2018) }\end{array}$ & Biospy & WB & $74 / 151$ & $104 / 136$ & $17 / 151$ & $22 / 136$ & $48 / 151$ & $10 / 136$ & $12 / 151$ & $0 / 136$ \\
\hline Feng et & & & - & - & - & - & $42 / 50$ & $25 / 54$ & - & - \\
\hline $\begin{array}{c}\text { al. } \\
(2015)\end{array}$ & Biospy & WB & - & - & - & - & $914 / 1109$ & $878 / 2052$ & - & - \\
\hline $\begin{array}{l}\text { Cui et al. } \\
\text { (2017) }\end{array}$ & Biospy & WB & - & - & - & - & $17 / 100$ & $33 / 54$ & - & - \\
\hline $\begin{array}{r}\text { Aver } \\
\text { fre }\end{array}$ & $\begin{array}{l}\text { ge weigl } \\
\text { quency }\end{array}$ & & $49 \%$ & $76 \%$ & $11 \%$ & $16 \%$ & $74 \%$ & $37 \%$ & $8 \%$ & $0 \%$ \\
\hline
\end{tabular}

Note: SCS: Source of cancer samples; SC: source of control; WB: wash brush; “_”: not data Source: The researcher's data analysis

Overall, the average weight frequency of RPMS1-A was $49 \%$ and $76 \%$ in NPC samples and non-cancerous samples; the average weight frequency of RPMS1-B was $11 \%$ and $16 \%$ in NPC samples and non-cancerous samples. Meanwhile, the frequencies of RPMS1-C variant were quite high in NPC samples (74\%) and low in non-NPC samples (34\%). In contrast to RPMS1-C, the variant of RPMS1-D appears with low frequency in both case and control samples ( $8 \%$ and $0 \%$, respectively).

According to A73, after exclusion studies that did not meet the inclusion criteria, up to now, only four studies carried out for analysis of polymorphism of $A 73$ gene in NPC. The characteristics of those studies were shown in Table 3. 
Table 3

Studies included in the systematic review about $A 73$ variant (A157154C) in NPC

\begin{tabular}{|c|c|c|c|c|c|c|c|c|}
\hline \multirow{2}{*}{ Studies } & \multirow{2}{*}{ SCS } & \multirow{2}{*}{ SC } & \multicolumn{2}{|c|}{$\mathbf{A A}$} & \multicolumn{2}{|c|}{ AC } & \multicolumn{2}{|c|}{$\mathrm{CC}$} \\
\hline & & & Case & Control & Case & Control & Case & Control \\
\hline $\begin{array}{c}\text { Shen et al. } \\
\text { (2015) }\end{array}$ & PB & PB & $54 / 510$ & $98 / 520$ & $214 / 510$ & $208 / 520$ & $242 / 510$ & $214 / 520$ \\
\hline $\begin{array}{c}\text { Zhang et al. } \\
\text { (2007) }\end{array}$ & Biospy & PB & $15 / 162$ & $24 / 99$ & $0 / 162$ & 9/99 & $147 / 1162$ & $66 / 99$ \\
\hline $\begin{array}{c}\text { Li et al. } \\
(2006)\end{array}$ & Biospy & WB & $0 / 7$ & - & $0 / 7$ & - & $7 / 7$ & - \\
\hline Han (2013) & Biospy & WB & $2 / 51$ & $20 / 52$ & $0 / 51$ & $10 / 52$ & $49 / 51$ & $22 / 52$ \\
\hline Average w & ht frequ & & $9 \%$ & $21 \%$ & $5 \%$ & $28 \%$ & $78 \%$ & $45 \%$ \\
\hline
\end{tabular}

Note: SCS: Source of cancer samples; SC: source of control; PB: peripheral blood; WB: wash brush; “-”: not data Source: The researcher's data analysis

In general, at nt 157154 in $A 73$ gene, the frequencies of CC genotype in NPC samples and non-cancerous samples were $78 \%$ and $45 \%$, respectively, higher than AA genotype (9\% in case, $21 \%$ in control) and AC genotype (5\% in case, $28 \%$ in control). The CC genotype frequency in the NPC group was significantly higher than that in the control group, the difference was significant (78\% vs. 45\%; p < 0.001). Therefore, A157154C polymorphism of the $A 73$ gene in EBV was associated with NPC susceptibility.

\subsection{Polymerase chain reaction (PCR) - sequencing method and selection primer}

The sequences of RPMS1 and $A 73$ genes was obtained from Genbank with the accession number NC_007605, V01555, respectively. The primers that identified variants in the RPMS1 genes were collected from the research by Feng et al. (2015). At the same time, primers identified variants in the $A 73$ gene were obtained from the study of Shen et al. (2015). Parameters of primer pairs were shown in Table 4 such as melting temperature, length, GCbase pair ratios, Gibbs Free Energy $(\Delta \mathrm{G})$ for secondary structures (hairpin, self-dimer, and heterodimer).

Table 4

Primer sequences and parameters

\begin{tabular}{|c|c|c|c|c|c|c|c|c|}
\hline \multirow{2}{*}{ Primer } & \multirow{2}{*}{ Sequence (5'-3') } & \multirow{2}{*}{$\mathbf{L}$} & \multirow{2}{*}{$\% \mathrm{GC}$} & \multirow{2}{*}{ Tm } & \multicolumn{3}{|c|}{$\Delta \mathbf{G}\left(\mathrm{Kcal} / \mathrm{mol}^{-1}\right)$} & \multirow{2}{*}{ (4) } \\
\hline & & & & & (1) & (2) & (3) & \\
\hline RPMS1-F & GCTGGGTTGATGCTGTAGATG & 21 & 52,4 & 55,9 & 0,32 & $-3,14$ & \multirow{2}{*}{$-3,17$} & \multirow{2}{*}{733} \\
\hline RPMS1-R & AGGGTCTGGACGTGGAGTTTG & 21 & 57,1 & 59,2 & 0,27 & $-6,3$ & & \\
\hline
\end{tabular}




\begin{tabular}{|c|c|c|c|c|c|c|c|c|}
\hline \multirow{2}{*}{ Primer } & \multirow{2}{*}{ Sequence (5'-3') } & \multirow{2}{*}{$\mathbf{L}$} & \multirow{2}{*}{$\% \mathrm{GC}$} & \multirow{2}{*}{$\mathbf{T m}$} & \multicolumn{3}{|c|}{$\Delta \mathrm{G}\left(\mathrm{Kcal} / \mathrm{mol}^{-1}\right)$} & \multirow{2}{*}{ (4) } \\
\hline & & & & & (1) & (2) & (3) & \\
\hline A73-F & AACATTGGGAATGAGAC & 17 & 41,2 & 43,6 & $\begin{array}{c}- \\
1,78 \\
\end{array}$ & $-5,37$ & \multirow{2}{*}{$-3,43$} & \multirow{2}{*}{711} \\
\hline A73-R & TTTGACCTGGAGGGCATC & 18 & 55,6 & 54,7 & $\begin{array}{c}- \\
1,14 \\
\end{array}$ & $-4,67$ & & \\
\hline
\end{tabular}

Note: L: Length of primer (bps); Tm: melting temperature $\left({ }^{\circ} \mathrm{C}\right)$; \%GC: GC-base pair ratios; Gibbs free energy $\left(\mathrm{Kcal}_{\mathrm{mole}}{ }^{-1}\right)$ for hairpin loop (1); homodimer (2) and heterodimer (3) structure formations; (4) Product sizes (bps).

Source: The researcher's data analysis

The primer was obtained from the study of Shen et al. (2015), used to amplify the sequence of $A 73$ gene by PCR method. The amplification product of the $A 73$ gene was shown in Figure 1.

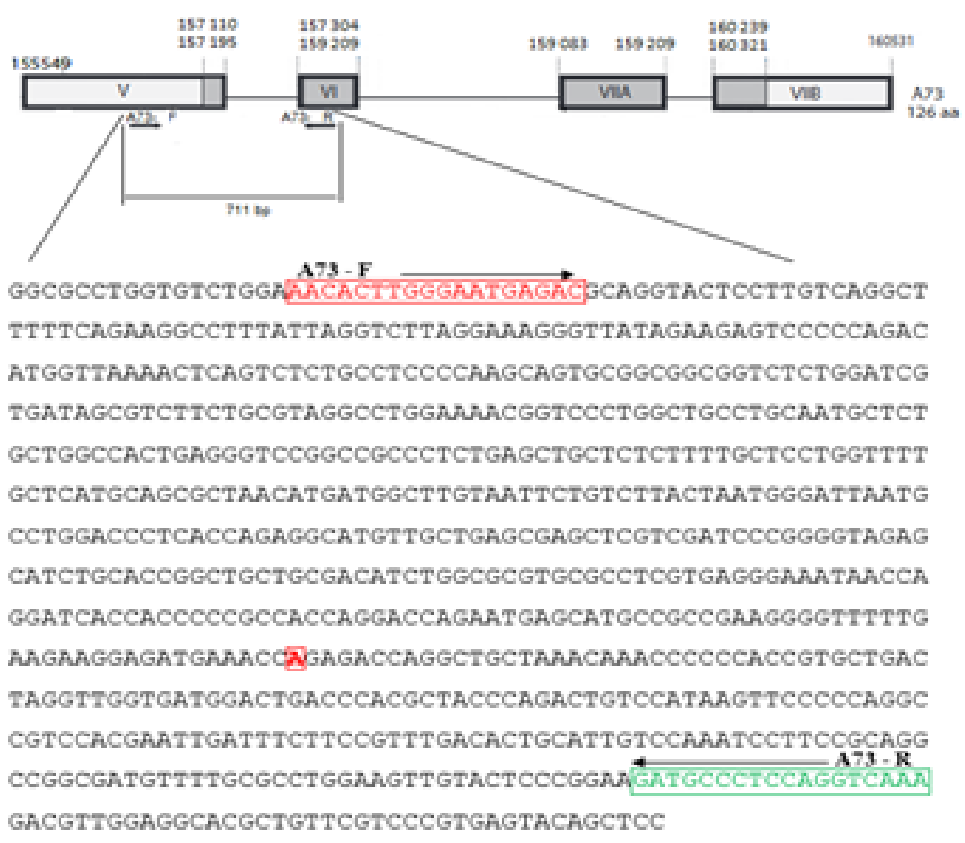

Figure 1. Position of primers used to amplified $A 73$ gene, and the location of SNPs

Note: The red nucleotide is located at polymorphism point 157154 on the strain B95-8 sequence (Accession number: V01555). The Figure 1 showed the position on the genome, structure of the $A 73$ gene and location of primers. A73 gene is described by exon (box) and intron (straight line). The open reading frame (ORF) of A73 genes was also shown as a dark gray box. The number of amino acids in the open reading frame was shown in the figure. Specific primer pairs were displayed by arrows and size of PCR products were also shown

In 2015, Feng et al. used SNP-RPMS1-F, R primer to conduct a position analysis of G155391A variant on NPC biopsy samples and benign brush samples in the Chinese population. Most recently in 2018, Wu et al. had also used this primer in their study to detect G155391A and other SNP variants of RPMS1 from NPC tissue samples and negative NPC brush samples. The amplification product of the primers, containing the sequence of variants in RPMS1 gene, was shown in Figure 2. 


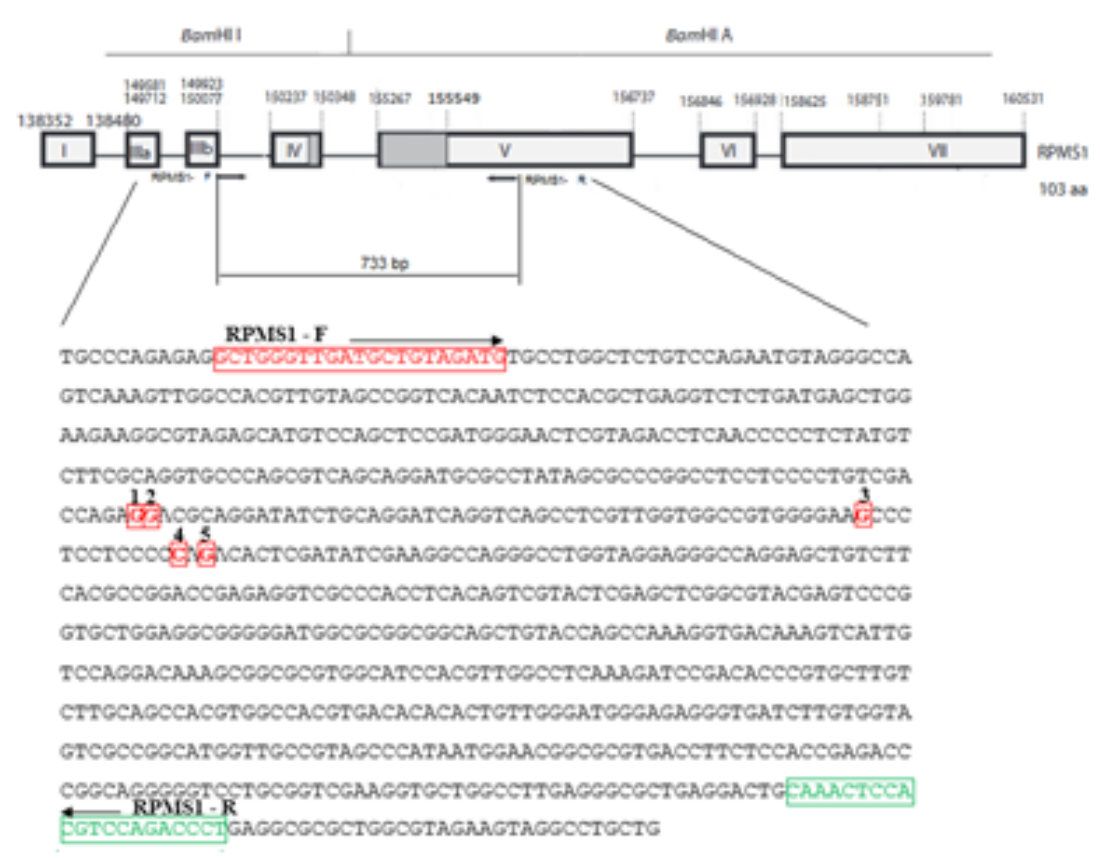

Figure 2. Position of primers used to amplify RPMS1 gene, and the location of SNPs Note: SNP at positions 155325, 155326, 155377, 155389 and 155391 on the Wild-type EBV sequence (Accession number: NC_007605). The Figure 2 shows the position on the genome, the structure of RPMS1 gene and primers. $R P M S 1$ is described by exons (box) and intron (straight line). The open reading frame RPMS1 was also shown as a dark gray box. The number of amino acids of $A 73$ protein and sizes of PCR products was also shown. Specific primer pairs were displayed by arrows

The sequences of RPMS1 and A73 genes were obtained from different accession numbers based on the distinguished origin of strains or types of infections. These sequences were conducted to align for compare sequences, the results were shown in Figures $3 \& 4$.

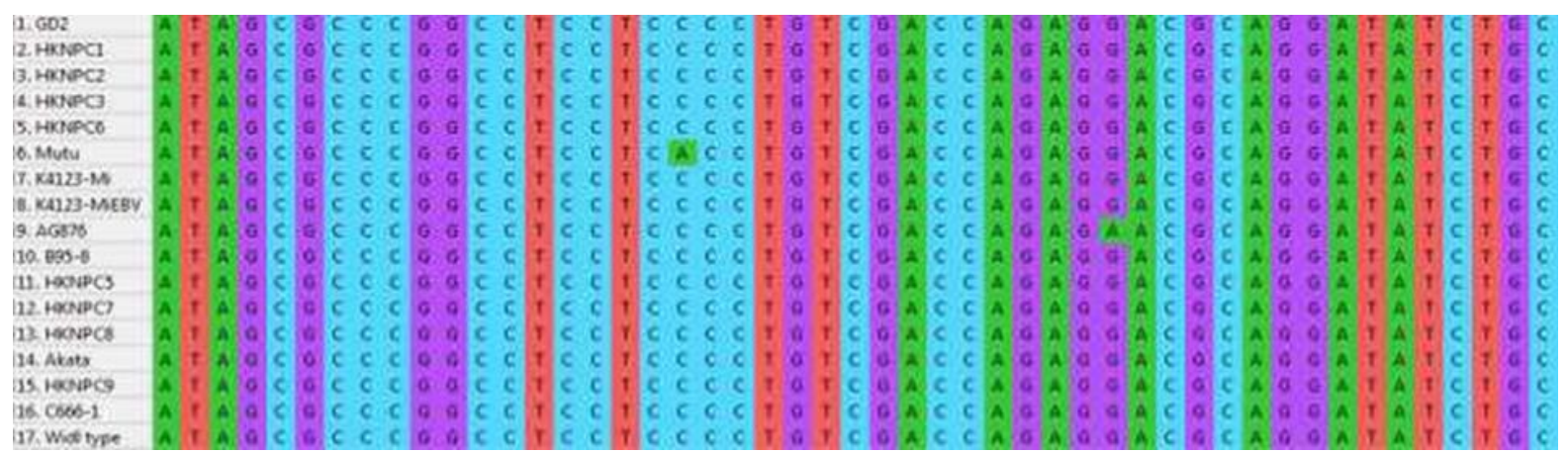

Figure 3. The sequence alignment of RPMS1 sequences from different strains 


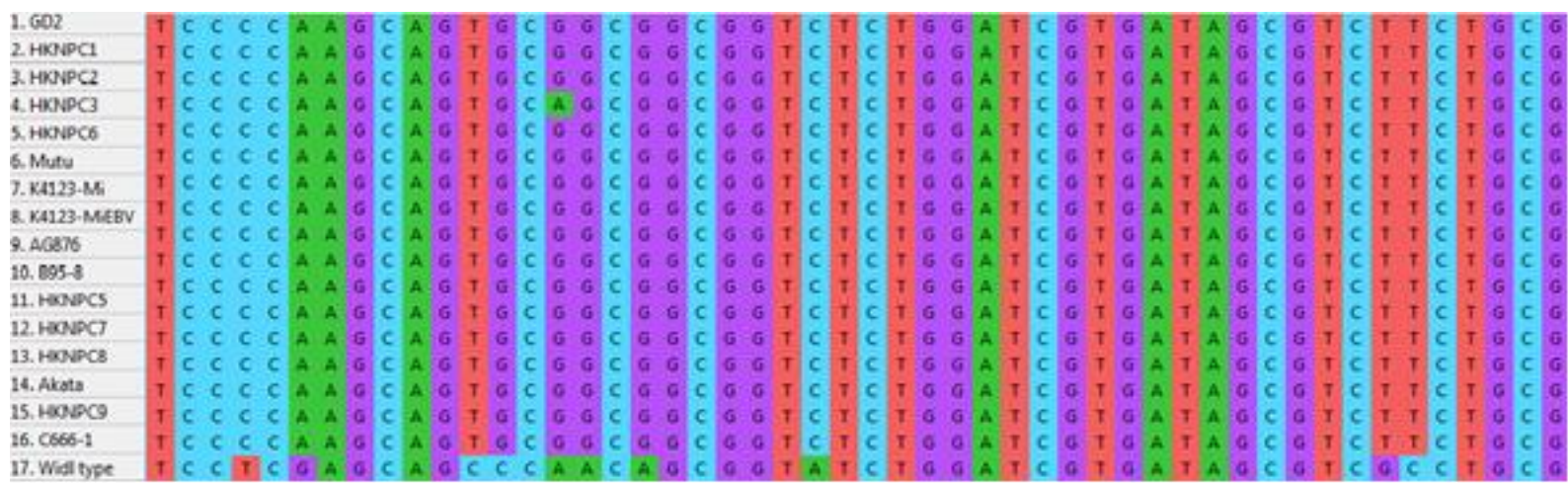

Figure 4. The sequence alignment of $A 73$ sequences from different strains

The results of the sequence alignment of RPMSI and A73 showed that the conservational ratio of this gene is quite high. In addition, the conservation sequences of this gene are very specific to EBV via BLAST (data not shown). Therefore, it can be concluded that the conservational gene region of $R P M S 1$ and $A 73$ has sufficient basis for the target sequences to analyze polymorphism in RPMS1 and $A 73$ genes.

\subsection{Phylogenetic tree of RPMS1 and A73 gene}

With the aim of surveying of the distribution of variants of RPMS1 and A73 gene in EBV strains in the world, thus, the database was collected from RPMSI and $A 73$ gene sequences of EBV belonging to different strains in the world, such as Asia (China, Hong Kong, Japan), Africa (Kenya, Ghana), America (USA) was collected to build phylogenetic trees. The database of RPMS1 and A73 gene included: GD1, GD2, HKNPC1, HKNPC2, HKNPC3, HKNPC4, HKNPC5, HKNPC6, HKNPC7, HKNPC8, HKNPC9, Akata, C666-1, Mutu, K4123-Mi, K4123-MiEBV, AG876, B95-8, Wild type, Macacine.

\section{Table 5}

Database of RPMS1 and $A 73$ gene

\begin{tabular}{|c|c|c|c|c|c|c|}
\hline STT & $\begin{array}{c}\text { Accession } \\
\text { number }\end{array}$ & Strain & $\begin{array}{c}\text { Subtype } \\
\text { (RPMS1) }\end{array}$ & $\begin{array}{c}\text { Subtype } \\
\text { (A73) }\end{array}$ & \multicolumn{2}{|c|}{ Country/ region } \\
\hline 1 & AY961628 & GD1 & RPMS1-B & - & China & \multirow{8}{*}{ Asia } \\
\hline 2 & HQ020558 & GD2 & RPMS1-C & A157154C & China & \\
\hline 3 & JQ009376 & HKNPC1 & RPMS1-C & A157154C & Hong Kong & \\
\hline 4 & KF992564 & HKNPC2 & RPMS1-C & A157154C & Hong Kong & \\
\hline 5 & KF992565 & HKNPC3 & RPMS1-C & A157154C & Hong Kong & \\
\hline 6 & KF992567 & HKNPC4 & RPMS1-C & A157154C & Hong Kong & \\
\hline 7 & KF992567 & HKNPC5 & RPMS1-C & A157154C & Hong Kong & \\
\hline 8 & KF992568 & HKNPC6 & RPMS1-C & A157154C & Hong Kong & \\
\hline
\end{tabular}




\begin{tabular}{|c|c|c|c|c|c|}
\hline STT & $\begin{array}{c}\text { Accession } \\
\text { number }\end{array}$ & Strain & $\begin{array}{c}\text { Subtype } \\
\text { (RPMS1) }\end{array}$ & $\begin{array}{c}\text { Subtype } \\
\text { (A73) }\end{array}$ & \multicolumn{2}{|c|}{ Country/ region } \\
\hline 9 & KF992569 & HKNPC7 & RPMS1-C & A157154C & Hong Kong \\
\hline 10 & KF992570 & HKNPC8 & RPMS1-C & A157154C & Hong Kong \\
\hline 11 & KF992571 & HKNPC9 & RPMS1-C & A157154C & Hong Kong \\
\hline 12 & KC207813 & Akata & RPMS1-B & - & Japan \\
\hline 13 & KC617875 & C666-1 & RPMS1-C & A157154C & Hong Kong \\
\hline 14 & KC207814 & Mutu & RPMS1-B & - & Kenya \\
\hline 15 & KC440851 & K4123-Mi & RPMS1-C & - & USA \\
\hline 16 & KC440852 & K4123- & - & - & USA \\
\cline { 1 - 5 } Of Asia \\
\hline 17 & DQ279927 & AG876 & RPMS1-D & - & Ghana \\
\hline 18 & V01555 & B95-8 & - & - & USA \\
\hline 20 & NC_007605 & Wild type & - & - & \\
\hline
\end{tabular}

Note: "-": The sequence has not yet detected variant.

Source: The researcher's data analysis

Phylogenetic tree of RPMS1 gene was built, based on the local database, aligned and synchronized. The length of the sequences after synchronization, was $733 \mathrm{bps}$, started from the nucleotide coding for amino acids 10 to amino acid 103 in each sequence. 


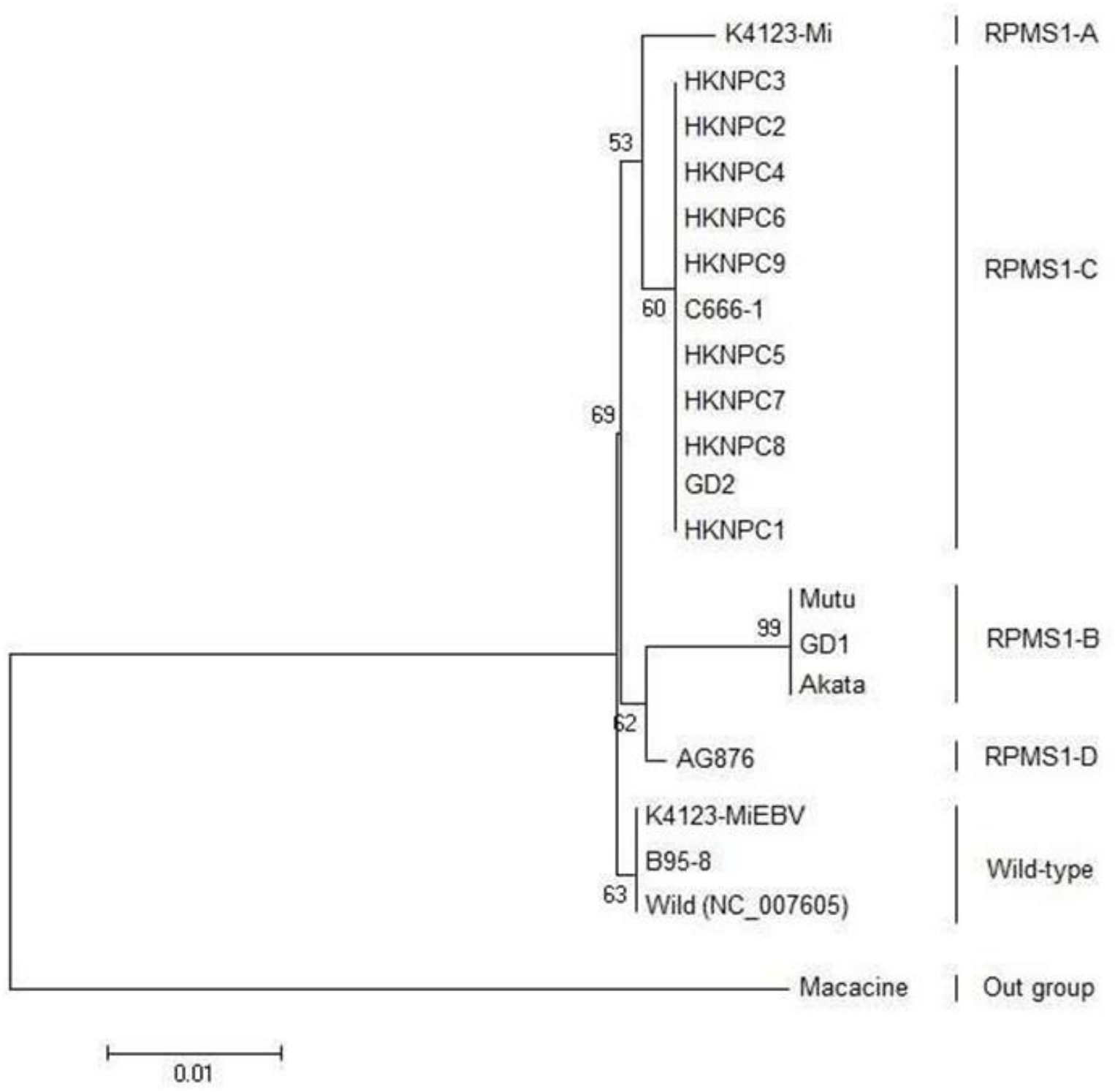

Figure 5. Phylogenetic tree of RPMS1 gene

In the phylogenetic tree, 19 nucleotide sequence of EBV strains were classified into four genotypes: RPMS1-A, RPMS1-B, RPMS1-C, and RPMS1-D. Line segment lengths proportional to the evolutionary distance of EBV strains. The results showed that RPMS1-C was detected in most of EBV strains in the world. These strains are mostly in Asia, such as HKNPC1, 2, 3, ... of Hong Kong, GD2 of China. The RPMS-B, RPMS1-A, RPMS1-D variant was detected less and scattered in different regions of the world. For example, RPMS1-D was detected in Ghana (Europe). The remaining variants (RPMS1-B, C, D) are variants that appeared with low frequency or are characteristic geographic variants.

Phylogenetic tree of $A 73$ gene was built, based on the local database, aligned and synchronized. The length of the sequences after synchronization, was $711 \mathrm{bps}$, started from the nucleotide coding for amino acids 1 in each sequence. 


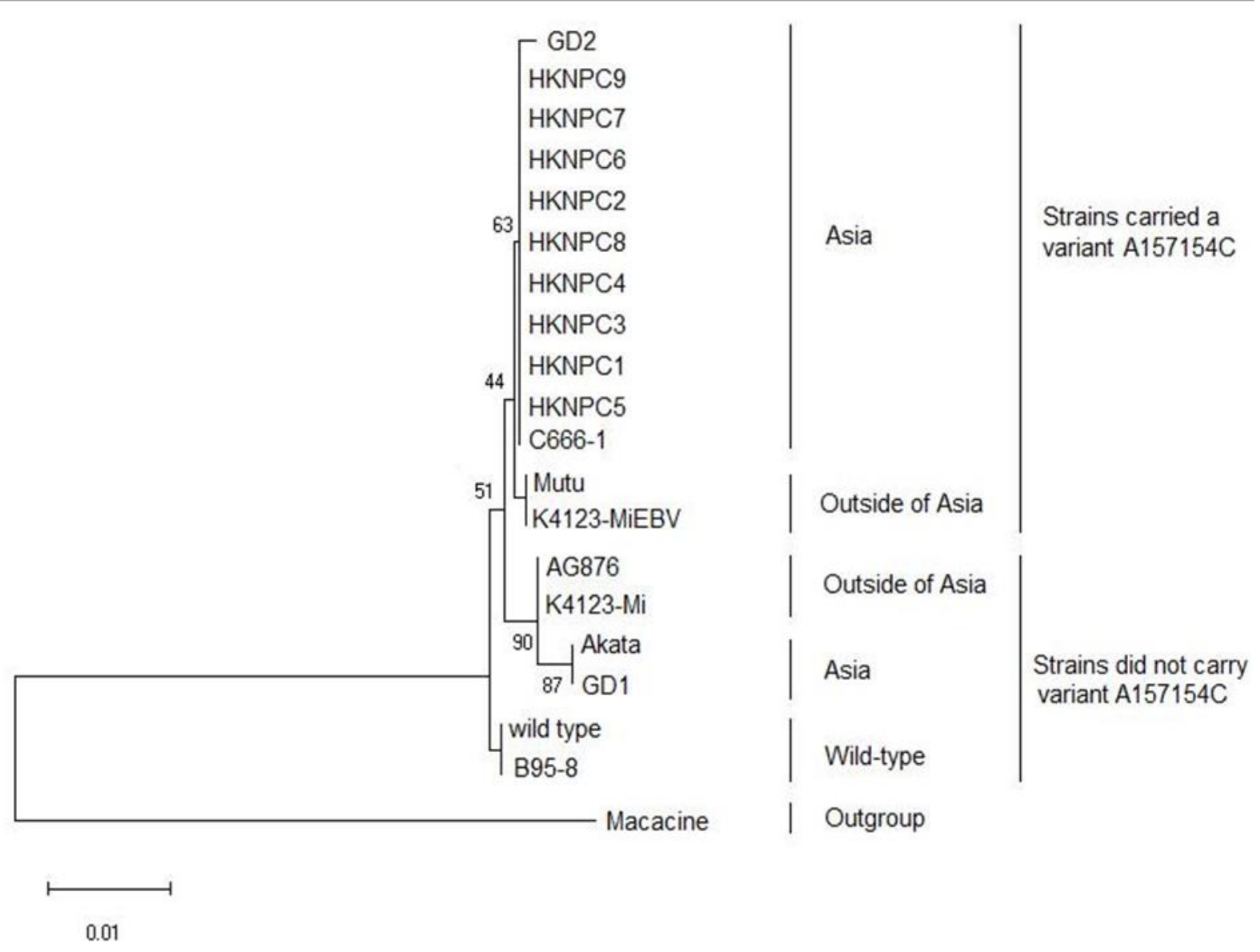

Figure 6. Phylogenetic tree of $A 73$ gene

The results of the phylogenetic tree of $A 73$ gene showed that the $\mathrm{A} 157154 \mathrm{C}$ variant was detected in most strains from Asia (C666-1, GD2, HKNPC1, etc.) and a few strains in Africa (Mutu), America (K4123-Mi). The EBV strains group did not carry out A157154 variant was relatively less than the group that carried A157154C variant, these strains were scattered in different regions of the world such as Japan (Akata), China (GD1), and the United States (K4123-Mi), Ghana (AG876). Consequently, the A157154C variant was mainly detected in EBV strains from Asia (especially Hong Kong, China), might be significantly associated with NPC in Asia - an endemic area for NPC.

\section{Discussion}

Nasopharyngeal carcinoma is one of the commonly occurring cancers among Asian region, including Vietnam with a high prevalence. Within the unclear symptoms, nasopharyngeal cancer often presents in the last stage when the first diagnosis. Therefore, the detection of NPC at the early stage is based on a potential biomarker (Epstein, 1993). For the past few years, in addition to the viral infections, which has been indicated certainly will lead to improve treatment and outcome for early diagnosis to be strongly related to human NPC, the polymorphism of RPMS1 and A73, is now regarded as one of the important mechanisms in the cancer development. In Vietnam, up to date, there was no study was conducted on the evaluation of an association between patterns of RPMS1 and A73 led to the risk of NPC. 
Therefore, in our initial study, we have to integrate the previous knowledge and findings to have an SNPs profile of RPMS1 and A73 genes by analyzing frequencies of RPMS1 and A73 variants.

According to a study by Wu et al. (2018), RPMS1-C and RPMS1-D exhibited a strong association with tumor formation. RPMS1-A could be detrimental to tumor formation, and RPMS1-B2 had a strong correlation with tumor formation in northern China. However, the mechanism that affects tumor formation related to these variants has not been clarified.

In the remaining studies of variants in RPMS1, these studies focused on analysis of the polymorphism point (G155391A) in RPMS1-C variants (Cui et al., 2017; Feng et al., 2015). The results show that this SNP position is a characteristic variant for NPC, especially in Cui et al. (2017), the frequencies of G155391A were significantly higher in the 50 matched samples from NPC patients (84\% in NPC biopsy samples and $82 \%$ in NPC washing brush samples) than that in 54 healthy throat washing samples (39\%) (Cui et al., 2017). Consequently, G155391A variant was determined significantly associated with NPC.

In the study of Feng et al. (2015), the authors indicated that the variant of G155391A at polymorphism point 155391 , changed $\mathrm{G} \rightarrow \mathrm{A}$, led to aa changed (Asp $\rightarrow$ Asn), which could be related to the transcription or expression of RPMS1. The function of SNP G155391A in RPMS1 has been proven in Feng's study. RPMS1 genes carried a variant $155391 \mathrm{~A}$ and did not carry variant (EBV wild-type) was grafted into pBABE-Puro vector. Then, these vectors were transferred and cultured on NP69 cell lines. Protein expression in these cells was examined by the Western blot method. Results showed that in cells treated with Cycloheximide (CHX) (which inhibited protein formation in eukaryote organisms), the degradation of RPMS 1 protein were observed after 0.5 hours in NP69 cells with wild-type RPMS1 (155391G), while the degradation of protein in NP69 cells with mutated RPMS1 (155391A) was slower (2.5 hours). At the same time, the half-life of the mutant RPMS1 protein 155391A was significantly longer than the RPMS1 protein without the mutation (3.2 vs $0.6 \mathrm{~h}$ ), indicating that SNP G155391A has a stabilizing function in RPMS1 protein or the stability of RPMS1 protein corresponds to the stability of the functional tumorigenesis.

Statistical analysis of $A 73$ variant showed that mutation at polymorphism point (A157145C) was a common SNP of $A 73$ on NPC samples. The average weight frequencies of CC genotype on NPC samples were $80 \%$. In the healthy sample, the average weight frequencies of CC genotype were $45 \%$, lower than the frequencies found in cancer samples. Therefore, it can be seen that the CC genotype at position 157145 is characteristic of NPC. It is obvious that all various studies on the polymorphisms of RPMS1 and $A 73$ were concentrated in China - the country with the highest prevalence of NPC in the world. Therefore, based on our data, we concluded RPMS1 and $A 73$ variants were significant association and contribution to the risk of NPC, might serve as molecular targets for screening test for NPC, which was affirmed again based on our systematic literature analysis, especially, in further study, applied in Vietnamese population. Not only that, this study will be a basic detailed database, give a hand to contribute the polymorphism data of EBV gene in the world, along with providing necessary data on gene polymorphism EBV, the distribution (epidemiology) of EBV strains in Vietnam. Additionally, based on molecular phylogenetic analysis, in further study, we could proceed to determine the 
relationship between polymorphism of RPMS1 and A73, identified in Vietnamese population, with the world.

\section{Conclusion}

We successfully performed a systematic literature revision and computed average detection frequencies of $R P M S 1$ and $A 73$ variants. A significant association between RPMS1C, RPMS1-D in RPMS1 gene; A157154C polymorphism of the A73 gene and NPC was shown and confirmed by systematical of previous studies. The location of variants were carried out and definitely confirmed the specificity and referent PCR primers. These databases will be the useful information for the identification of variants in RPMS1 and $A 73$ genes that may serve as biomarker for the further experiment and screening test for NPC in Vietnamese population.

\section{References}

Brooks, L. A., Lear, A. L., Young, L. S., \& Rickinson, A. B. (1993). Transcripts from the Epstein-Barr virus BamHI A fragment are detectable in all three forms of virus latency. Journal of Virology, 67(6), 3182-3190.

Cui, Q., Feng, F. T., Xu, M., Liu, W. S., Yao, Y. Y., Xie, S. H., ... Bei, J. X. (2017). Nasopharyngeal carcinoma risk prediction via salivary detection of host and Epstein-Barr virus genetic variants. Oncotarget, 8(56), 95066-95074.

de Jesus, O., Smith, P. R., Spender, L. C., Karstegl, C. E., Niller, H. H., Huang, D., \& Farrell, P. J. (2003). Updated Epstein-Barr Virus (EBV) DNA sequence and analysis of a promoter for the BART (CST, BARF0) RNAs of EBV. Journal of General Virology, 84(6), 1443-1450.

Epstein, J. B., \& Jones, C. K. (1993). Presenting signs and symptoms of nasopharyngeal carcinoma. Oral Surgery, Oral Medicine, Oral Pathology, and Oral Radiology, 75, 3236.

Feng, F. T., Cui, Q., Liu, W. S., Guo, Y. M., Feng, Q. S., Chen, L. Z., ... Middeldorp, J. M. (2015). A single nucleotide polymorphism in the Epstein-Barr virus genome is strongly associated with a high risk of nasopharyngeal carcinoma. Chinese Journal of Cancer, 34(3), 61-71.

GLOBOCAN. (2018). Estimated cancer incidence, mortality and prevalence worldwide in 2018 Retrieved March 30, 2019, from http://globocan.iarc.fr/Pages/fact_sheets_population.aspx.

Han, R. M. (2013). Research progress in the CDKN2A gene and EBV A73 gene Polymorphism with Nasopharyngeal Carcinoma in Yunnan China. Retrieved March 30, 2019, from Globe Thesis website: https://www. globethesis.com/?t=2214330374955373

Hildesheim, A., \& Levine, P. H. (1993). Etiology of nasopharyngeal carcinoma: A review. Epidemiologic Reviews, 15(2), 466-485.

Li, A., Zhang, X. S., Jiang, J. H., Wang, H. H., Liu, X. Q., Pan, Z. G., \& Zeng, Y. X. (2005). Transcriptional expression of RPMS1 in nasopharyngeal carcinoma and its oncogenic potential. Cell Cycle, 4(2), 303-308. 
Li, A., Zhao, S., Hu, W., Zhao, G. F., \& Zhang, X. S. (2006). Cloning of A73 gene and its coding sequence analysis. Journal of Southern Medical University, 26(6), 826-830.

Regaud, C. (1921). Lympho-épitheliome de l'hypopharynx traité par la roentgenthérapie. Bull Soc Franc Otorhinolaryngol, 34, 209-214.

Sadler, R. H., \& Raab-Traub, N. (1995). Structural analyses of the Epstein-Barr virus BamHI A transcripts. Journal of Virology, 69(2), 1132-1141.

Schmincke, A. (1921). Uber lymphoepitheliale geschwulste. Beitr Path Anat, 78, 161-170.

Shen, J. J., Niu, W. N., Zhou, M., Zhou, F., Zhang, H. Y., \& Wang, L. (2015). Association of Epstein Barr virus A73 gene polymorphism with nasopharyngeal carcinoma. Genetic Testing and Molecular Biomarkers, 19(4), 187-190.

Smith, P. R., De Jesus, O., Turner, D., Hollyoake, M., Karstegl, C. E., Griffin, B. E., ... Farrell, P. J. (2000). Structure and coding content of CST (BART) family RNAs of Epstein-Barr virus. Journal of Virology, 74(7), 3082-3092.

Wu, S., Liu, W., Li, H., Zhao, Z., Yang, Y., Xiao, H., ... Luo, B. (2018). Conservation and polymorphism of EBV RPMS1 gene in EBV-associated tumors and healthy individuals from endemic and non-endemic nasopharyngeal carcinoma areas in China. Virus research, 250, 75-80.

Zhang, L. L., Li, D. J., Li, Z. H., Zhang, X. S., Zhang, R. H., Yu, X. J., ... Jia, W. H. (2007). Correlation of Epstein-Barr virus A73 gene polymorphisms to susceptibility to nasopharyngeal carcinoma. Chinese Journal of Cancer, 26(10), 1047-1051. 